\title{
Clinical significance of ALDH1 combined with DAPI expression in patients with esophageal carcinoma
}

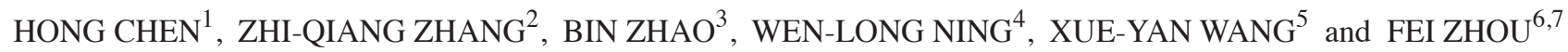 \\ ${ }^{1}$ Center of Individualized Medicine Diagnosis and Treatment, The First Hospital of Qiqihar, Qiqihar, Heilongjiang 161005; \\ ${ }^{2}$ Department of First General Surgery, The First Hospital of Harbin, Harbin, Heilongjiang 150010; ${ }^{3}$ Department of \\ Anorectal Surgery, The First Hospital Affiliated of Jiamusi University, Jiamusi, Heilongjiang 154000; Departments of \\ ${ }^{4}$ Emergency, ${ }^{5}$ Public Health and ${ }^{6}$ Second General Surgery, The First Hospital of Qiqihar, Qiqihar, Heilongjiang 161005; \\ ${ }^{7}$ Harbin Medical University Clinical Post-Doctor, Harbin, Heilongjiang 150001, P.R. China
}

Received November 21, 2015; Accepted May 8, 2017

DOI: $10.3892 / 01.2017 .6743$

\begin{abstract}
Esophageal carcinoma is the most common type of tumor, with the incidence in China accounting for $50 \%$ of cases worldwide and the majority of patients not surviving due to tumor recurrence. According to the cancer stem cell theory, tumor development and recurrence is due to the excitation of a cancer stem cell. Aldehyde dehydrogenase 1 (ALDH1) is an appropriate marker for cancer stem cells and the present study aimed at determining the function of ALDH1 in human esophageal carcinoma. Indirect fluorescence antibody staining was used to investigate the association between the level of ALDH1 protein expression and clinicopathological parameters, including sex, age, vein invasion, degree of tumor cell differentiation and clinical stage. DAPI was used to stain the nuclei of tumor cells and exclude non-tumor cells. The results of the present study revealed that ALDH1 expression was associated with the level of tumor cell differentiation, tumor-node-metastasis stage and lymphatic invasion. In addition, increased expression of ALDH1 was identified in esophageal carcinoma tissues compared with in healthy esophageal tissues. Therefore, ALDH1 may be used as a parameter for the pathology of esophageal carcinoma.
\end{abstract}

\section{Introduction}

Esophageal carcinoma is the most common type of cancer worldwide with $\sim 482,300$ novel cases and 406,800 mortalities reported annually (1). In China, the incidence of esophageal carcinoma accounts for $50 \%$ of cases worldwide

Correspondence to: Dr Fei Zhou, Department of Second General Surgery, The First Hospital of Qiqihar, 30 Park Road, Longsha, Qiqihar, Heilongjiang 161005, P.R. China

E-mail: zhoufei789@outlook.com

Key words: aldehyde dehydrogenase 1, DAPI, human esophageal carcinoma, cancer stem cell, stem cell, indirect fluorescent immunostaining and esophageal carcinoma is the fourth leading cause of malignant tumor-associated mortality, with $95 \%$ of esophageal cancer cases diagnosed as squamous cell carcinoma (2). Surgery is the optimal treatment for patients with esophageal carcinoma $(3,4)$; however, the majority of patients do not survive due to tumor recurrence, in spite of radical resection and extended lymph node dissection having been performed. A number of factors affect tumor recurrence including age, sex, local tumor stage, tumor location, degree of cell differentiation, lymph node metastases or vascular involvement (5).

Aldehyde dehydrogenase 1 (ALDH1) is a detoxifying enzyme which responds to the oxidation of intracellular aldehydes $(6,7)$. The function of ALDH1 is to oxidize intracellular aldehydes and therefore confer resistance to alkylating agents (8). As a modulator of cell viability, ALDH1 converts retinol into retinoic acid, which serves an important function in the early differentiation of stem cells (9). Murine, human hematopoietic, neural stem and progenitor cells have been identified to exhibit increased ALDH1 activity, and ALDH1 activity is a commonly used marker for healthy and malignant stem cells $(10,11)$. Previous immunohistochemistry results have demonstrated that ALDH1 expression was limited in healthy tissue, but was markedly increased in malignant tissue, including breast, lung and colorectal cancer (12-14). However, whether the expression of ALDH1 is associated with the differentiation of tumor cells in esophageal carcinoma remains unknown. Therefore, the present study aimed at identifying whether ALDH1 expression exhibited an association with patients with esophageal cancer using fluorescent immunostaining.

In 1994, Grimason et al (15) used the fluorogen DAPI to interact with the nuclei of sporulated oocysts in conjunction with a fluorescein isothiocyanate-conjugated anti-cryptosporidium monoclonal antibody and used fluorescence microscopy to visualize the oocyst nuclei, which enabled improved observation. DAPI is a non-cytotoxic dye that does not affect cell viability (16). DAPI is able to be combined with cellular DNA, permeate through the membrane of cell, rapidly enter the nucleus of living cells and bind with DNA to form a DAPI-DNA complex. The wavelengths of the complex for excitation and emission are 360 and $460 \mathrm{~nm}$, respectively. 
Under the excitation of an ultraviolet ray, DAPI exhibits blue fluorescence, therefore under a fluorescence microscope a blue nucleus may be observed. The formula of DAPI is $\mathrm{C}_{16} \mathrm{H}_{15} \mathrm{~N}_{5}$ and the molecular mass is $277,324 \mathrm{Da}(17)$.

In the present study, DAPI was used to non-specifically stain the nuclei of the tumor cells. Subsequently, ALDH1-specific fluorescent staining was used on the cancer stem cell cytoplasm and a merged image was developed. The aim of the present study was to use indirect fluorescent immunostaining to identify whether the expression of ALDH1 may be a notable clinicopathological prognostic factor for human esophageal carcinoma.

\section{Materials and methods}

Patients and tissues. Specimens of human esophageal squamous cell carcinomas were obtained from the Department of Pathology, The First Hospital of Qiqihar (Qiqihar, China) between January 2010 and January 2014. Prior to surgery no patients had received any therapy, including chemotherapy or radiation. Of the 50 specimens, 10 cases were well-differentiated, 20 were moderately differentiated and 20 cases were poorly differentiated squamous tumor cells. In addition, healthy esophageal tissues were obtained from the same cohort of patients, but these were obtained from a distant location from the esophageal carcinoma $(\geq 5 \mathrm{~cm})$. All tissues (thickness, $4 \mu \mathrm{m}$ ) were fixed in formalin, embedded in paraffin. When required, the tissues were deparaffinized and dehydrated in $10 \%$ formalin at $27^{\circ} \mathrm{C}$ for $5 \mathrm{~min}$. The present study was approved by the Ethical Committee of the First Hospital of Qiqihar. Additionally, written informed consent was obtained from all participating patients. All tissues were evaluated by two pathologists individually.

Indirect fluorescent immunostaining. Indirect fluorescent immunostaining was performed as described previously (18). First, DAPI (dilution, 1:500; cat no. D9564; Sigma-Aldrich; Merck KGaA, Darmstadt, Germany) was used to non-specifically stain the nuclei of cancer cells (15). The cells were then incubated at $37^{\circ} \mathrm{C}$ with fluorophore I-labeled IgG (dilution, 1:20; catalog no. HZ3387121; excitation, 360 nm; emission, $460 \mathrm{~nm}$; EarthOx Life Sciences, Millbrae, CA, USA) for $30 \mathrm{~min}$. Sections were rinsed with TBS-Tween-20 (TBST) three times and incubated at $4^{\circ} \mathrm{C}$ with an antibody against ALDH1 (dilution, 1:400; cat no. HZ3487111; EarthOx Life Sciences) overnight. Subsequently, sections were incubated at $37^{\circ} \mathrm{C}$ with fluorophore II-labeled IgG (dilution, 1:50; cat no. HZ3387125; excitation, $490 \mathrm{~nm}$; emission, $520 \mathrm{~nm}$; EarthOx Life Sciences) for $30 \mathrm{~min}$. Sections were rinsed with TBST three times and coverslips were placed on the slides. Finally, fluorescence microscopy (magnification, x200; Nikon Eclipse 80i; Nikon Corporation, Tokyo, Japan) was used to observe the results.

Evaluation of labeling. Evaluation of the expression of ALDH1 was performed by two pathologists independently. Imaging analysis of ALDH1 expression was performed in one selected area per case. Cases exhibiting $\geq 20 \%$ positive cells were classified as significant and the remaining cases were classified as negative ALDH1 expression (19).
Table I. Patient characteristics.

\begin{tabular}{lc}
\hline Clinical data & $\mathrm{n}(\%)$ \\
\hline Total & 50 \\
Male & $33(66)$ \\
Female & $17(34)$ \\
Age, years & \\
$\geq 60$ & $27(54)$ \\
$<60$ & $23(46)$ \\
Median & $52.3(35-70)$ \\
Differentiation & \\
Well & $10(20)$ \\
Moderate & $20(40)$ \\
Poor & $20(40)$ \\
TNM stage & \\
I/II & $23(46)$ \\
III/IV & $27(54)$ \\
Lymph node metastasis & \\
Positive & $28(56)$ \\
Negative & $22(44)$ \\
Vein invasion & \\
Positive & $26(52)$ \\
Negative & $24(48)$ \\
\end{tabular}

Table II. Comparison between ALDH1-positive expression in esophageal cancer and healthy esophageal tissues.

\begin{tabular}{lcccc}
\hline Group & Total & ALDH1-positive, $\%$ & $\chi^{2}$ & P-value \\
\hline $\begin{array}{l}\text { Esophageal } \\
\text { cancer tissue }\end{array}$ & 50 & $23(46)$ & & \\
$\begin{array}{l}\text { Healthy } \\
\text { esophageal tissue }\end{array}$ & 50 & $8(16)$ & 5.259 & $<0.05$ \\
\hline
\end{tabular}

ALDH1, aldehyde dehydrogenase.

Statistical analysis. All data were analyzed using SPSS software (version 12.0; SPSS, Inc., Chicago, IL, USA). The association between the expression of ALDH1 and the clinicopathological parameters was evaluated using the $\chi^{2}$ test. In addition, the expression of ALDH1 between esophageal squamous cell carcinoma and healthy esophageal tissues were analyzed using the $\chi^{2}$ test. $\mathrm{P}<0.05$ was considered to indicate a statistically significant difference.

\section{Results}

Patient characteristics. In the present study, a total of 50 patients were included. A total of 33 were male $(66 \%)$ and 17 were female (34\%), a ratio of $1.94: 1$. The patient median age was 52.3 years (range, 35-70 years), with 27 (54\%) and $23(46 \%)$ patients aged $\geq 60$ and $<60$ years, respectively. All patients were diagnosed with human esophageal squamous 
Table III. Association between ALDH1-positive expression and the clinicopathological features of human esophageal carcinoma.

\begin{tabular}{|c|c|c|c|c|c|c|}
\hline Clinicopathological feature & $\mathrm{n}$ & Positive & Negative & Positive rate, $\%$ & $\chi^{2}$ & P-value \\
\hline Sex & & & & & & $>0.05$ \\
\hline Male & 33 & 13 & 20 & 39.4 & 1.705 & \\
\hline Female & 17 & 10 & 7 & 58.8 & & \\
\hline Age, years & & & & & & $>0.05$ \\
\hline$\geq 60$ & 27 & 13 & 14 & 48.1 & 0.109 & \\
\hline$<60$ & 23 & 10 & 13 & 43.5 & & \\
\hline Histological grade & & & & & & $<0.05$ \\
\hline Well & 10 & 2 & 8 & 20 & 11.554 & \\
\hline Moderate & 20 & 6 & 14 & 30 & & \\
\hline Poor & 20 & 15 & 5 & 75 & & \\
\hline TNM stage & & & & & & $<0.05$ \\
\hline I/II & 23 & 6 & 7 & 26.1 & & \\
\hline III/IV & 27 & 17 & 10 & 63.0 & 6.798 & \\
\hline Lymphatic invasion & & & & & & $<0.05$ \\
\hline Positive & 28 & 18 & 10 & 64.3 & 8.567 & \\
\hline Negative & 22 & 5 & 17 & 22.7 & & \\
\hline Vein invasion & & & & & & $>0.05$ \\
\hline Positive & 26 & 11 & 15 & 42.3 & 0.290 & \\
\hline Negative & 24 & 12 & 12 & 50.0 & & \\
\hline
\end{tabular}

ALDH1, aldehyde dehydrogenase; TNM, tumor-node-metastasis.

cell carcinoma, and classified as exhibiting well-, moderately or poorly differentiated tumor cells. A total of $10(20 \%), 20$ (40\%) and $20(40 \%)$ of patients exhibited well-, moderately and poorly differentiated tumor cells, respectively. In addition, patients were staged according to the tumor-node-metastasis (TNM) classification (20). A total of $23(46 \%)$ patients were at TNM stages I/II and 27 (54\%) were at stages III/IV. Lymphatic and vein invasion was observed in 28 (56\%) and $26(52 \%)$ patients, respectively (Table I).

Expression of ALDH1 in healthy esophageal tissues and esophageal carcinoma tissues. Expression of ALDH1 was identified in the cytoplasm of esophageal carcinoma tissues and a limited number of healthy esophageal tissues. Human esophageal carcinoma tissues exhibited markedly increased expression levels of ALDH1 protein, compared with that of healthy esophageal tissues. In addition, compared with healthy esophageal tissues, human esophageal carcinoma tissues exhibited significantly increased expression levels of ALDH1 protein $\left(\chi^{2}=5.259 ; \mathrm{P}<0.05\right)$. Of the 50 healthy controls, ALDH1 activity was identified in $\sim 16 \%$ of esophageal cells. However, in the 50 esophageal cancer tissues, positive ALDH1 incidence was $46 \%$. The results are presented in Table II.

Association between the expression of ALDHI and the clinicopathological features of esophageal carcinoma. First, DAPI was used to stain the cancer cell nuclei, which excluded non-tumor cells. The association between ALDH1 protein expression and the clinicopathological features of human esophageal squamous cell carcinoma are summarized in Table III. No significant difference was identified between the expression of ALDH1 and sex, age or vein invasion. However, ALDH1 expression was identified to be associated with the level of differentiation of the tumor cells. It was revealed that, as the level of differentiation of tumor cells decreased, the positive rate of ALDH1 expression increased. Compared with well- and moderately differentiated tumor cells (Figs. 1 and 2), the ALDH1 intensity was markedly increased in poorly differentiated malignant tumor cells (Fig. 3). All images were stained individually and finally merged. A positive association was identified between the differentiation of tumor cells and the positive expression of ALDH1 $\left(\chi^{2}=11.554 ; \mathrm{P}<0.05\right)$. ALDH1-positive expression was only observed in 2/10 (20\%), $6 / 20(30 \%)$ and $15 / 20(75 \%)$ of the well-, moderately and poorly differentiated cases, respectively. In addition, patients of stages III/IV esophageal carcinoma exhibited an increased expression rate of ALDH1 (63.0\%), compared with those of stages I/II $(26.1 \%)\left(\chi^{2}=6.789 ; \mathrm{P}<0.05\right)$. Furthermore, in the cases of lymphatic invasion, the positive rate of ALDH1 expression $(64.3 \%)$ was increased, compared with that of the cases without lymphatic invasion $\left(22.7 \% ; \chi^{2}=8.567 ; \mathrm{P}<0.05\right)$.

\section{Discussion}

Human esophageal cancer is a life-threatening disease worldwide. Although radical surgery may be performed, the 5-year survival rate rarely exceeds $30 \%$. A number of patients with the early-stage disease exhibit an increased risk of disease recurrence following treatment (21). Esophageal carcinomas are divided into the adenocarcinoma and squamous cell 

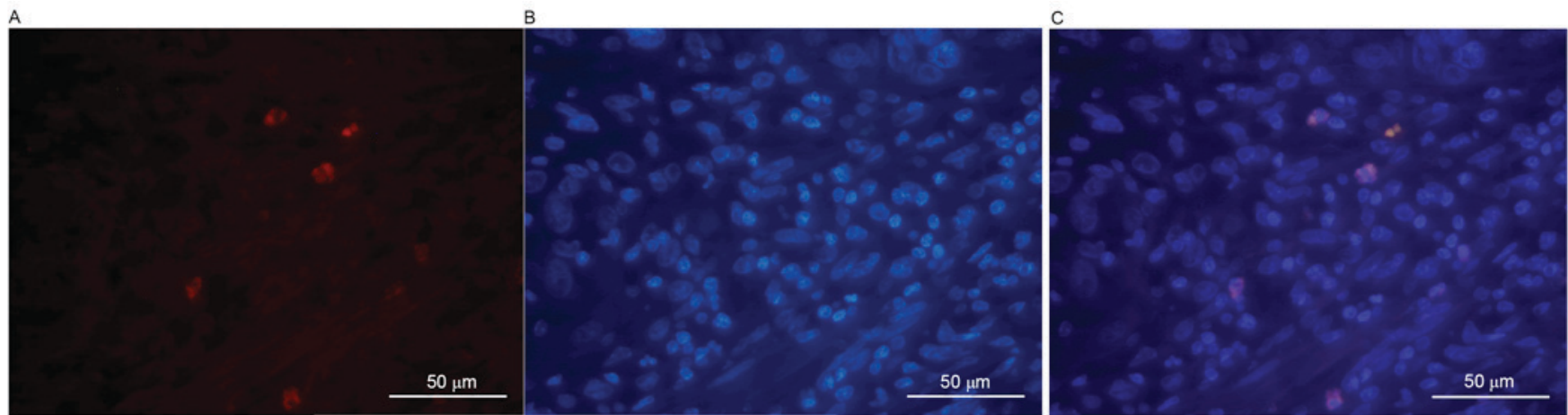

Figure 1. ALDH1 combined with DAPI expression in well-differentiated cells of esophageal carcinoma tissues. (A) ALDH1 expression. (B) DAPI expression. (C) Merged image of A and B (magnification, x200). ALDH1, aldehyde dehydrogenase.
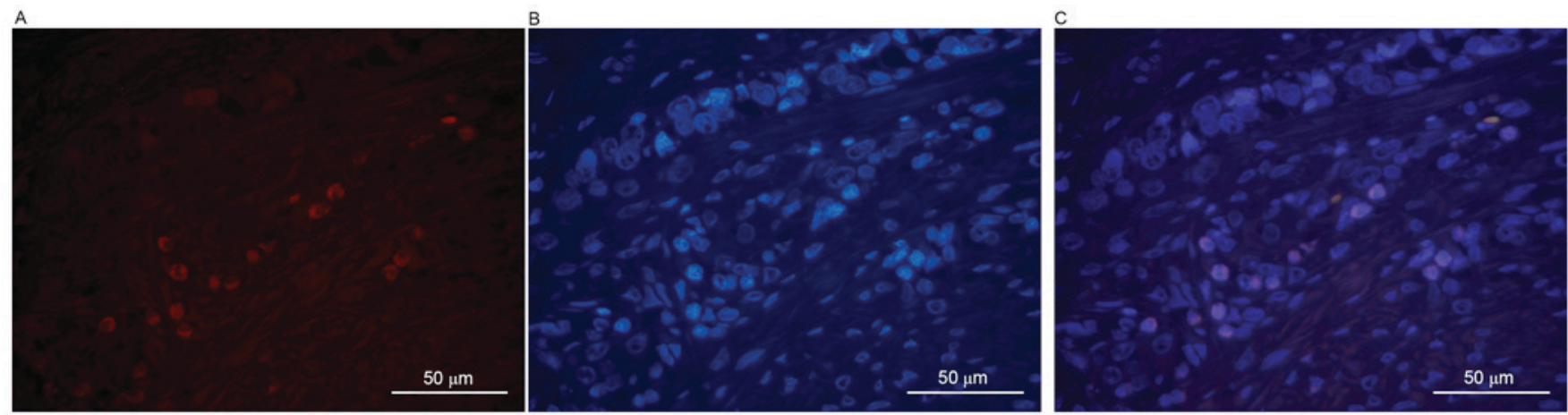

Figure 2. ALDH1 combined with DAPI expression in moderately differentiated cells of esophageal carcinoma tissues. (A) ALDH1 expression. (B) DAPI expression. (C) Merged image of A and B (magnification, x200). ALDH1, aldehyde dehydrogenase.
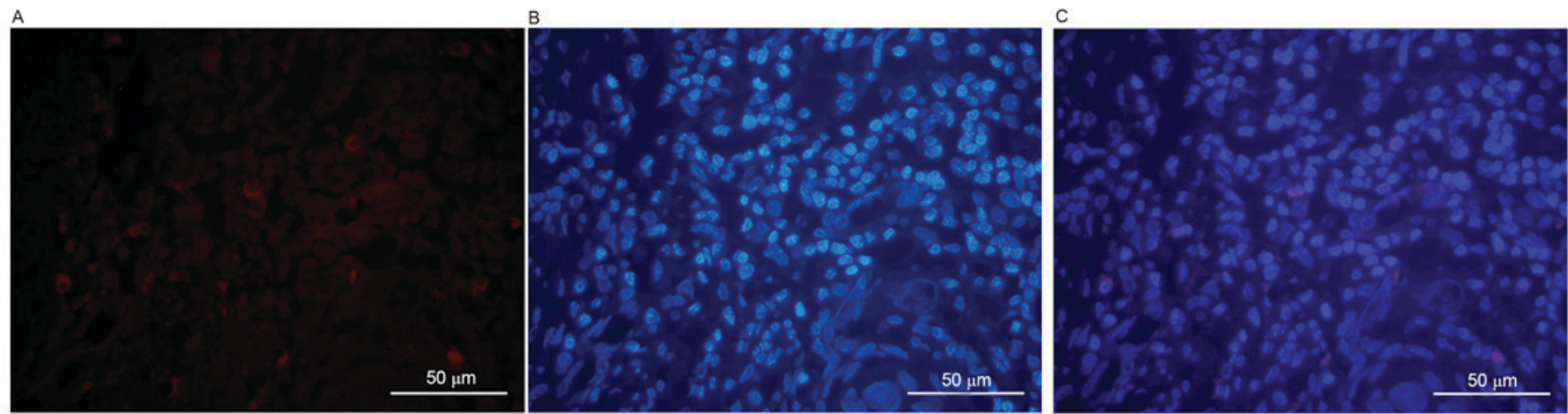

Figure 3. ALDH1 combined with DAPI expression in poorly differentiated cells of esophageal carcinoma tissues. (A) ALDH1 expression. (B) DAPI expression. (C) Merged image of A and B (magnification, x200). ALDH1, aldehyde dehydrogenase.

carcinoma histological subtypes; the latter accounts for the majority of esophageal carcinoma cases. Increased incidence of esophageal cancer is due to obesity, smoking, consumption of hot beverages and red meat, increased alcohol intake and a decreased intake of fresh vegetables or fruit (22). The morbidity of squamous cell carcinoma is increasing in developing countries, particularly in China (23).

According to the cancer stem cell theory, malignant tumors develop from a cancer stem cell, which has the capability of pluripotency and self-renewal (24). ALDH1, a detoxifying enzyme responsible for the oxidation of intracellular aldehydes, is a marker of cancer stem cells (10). The results of the present study demonstrated that, compared with healthy esophageal tissues, esophageal cancer tissues expressed an increased positive rate of $\operatorname{ALDH} 1\left(\chi^{2}=5.259\right.$; $\left.\mathrm{P}<0.05\right)$. In addition, an association between the differentiation degree of tumor cells and the expression of ALDH1 was identified. Using indirect immunofluorescence staining, it was demonstrated that an increased degree of differentiation of the tumor cells was associated with decreased expression of ALDH1 $\left(\chi^{2}=11.554 ; \mathrm{P}<0.05\right)$. Furthermore, TNM stages III/IV exhibited an increased positive expression of ALDH1, compared with that of TNM stages I/II $\left(\chi^{2}=6.789 ; \mathrm{P}<0.05\right)$. Additionally, it was identified that positive rates of ALDH1 expression were 
increased in cases of lymphatic invasion, compared with that in the tissues without lymphatic invasion $\left(\chi^{2}=8.567 ; \mathrm{P}<0.05\right)$. In the present study, DAPI was used to non-specifically stain the nuclei of tumor cells to exclude the impact of non-tumor cells.

The expression of ALDH1 was associated with the clinicopathological characteristics of human esophageal squamous cell carcinoma. ALDH1 may serve an important function in the process of human esophageal cancer. However, whether ALDH1 may be used alone to identify cancer stem cells and whether the prognosis of patients may be predicted on the basis of ALDH1 expression requires additional studies.

\section{References}

1. Jemal A, Bray F, Center MM, Ferlay J, Ward E and Forman D: Global cancer statistics. CA Cancer J Clin 61: 69-90, 2011.

2. Guo M, Zhao YD, Yang HJ and Yan XF: Analysis of clinicopathological characteristics for 5406 cases of esophageal neoplasm. Chin J Cancer Prev Treat 15: 54-56, 2008.

3. Debevec L, Jerič T, Kovač V, Bitenc M and Sok M: Is there any progress in routine management of lung cancer patients? A comparative analysis of an institution in 1996 and 2006. Radiol Oncol 43: 47-53, 2009.

4. Kovac V, Zwitter M and Zagar T: Improved survival after introduction of chemotherapy for malignant pleural mesothelioma in Slovenia: Population-based survey of 444 patients. Radiol Oncol 46: 136-144, 2012

5. Shi HY, Zhu SC, Shen WB and Liu ML: Pathological characteristics of esophageal cancer. Oncol Lett 8: 533-538, 2014.

6. Magni M, Shammah S, Schiró R, Mellado W, Dalla-Favera R and Gianni AM: Induction of cyclophosphamide-resistance by aldehyde-dehydrogenase gene transfer. Blood 87: 1097-1103, 1996.

7. Sophos NA and Vasiliou V: Aldehyde dehydrogenase gene superfamily: The 2002 update. Chem Biol Interact 143-144: 5-22, 2003.

8. Dylla SJ, Beviglia L, Park IK, Chartier C, Raval J, Ngan L, Pickell K, Aguilar J, Lazetic S, Smith-Berdan S, et al: Colorectal cancer stem cells are enriched in xenogeneic tumors following chemotherapy. PLoS One 3: e2428, 2008.

9. Chute JP, Muramoto GG, Whitesides J, Colvin M, Safi R, Chao NJ and McDonnell DP: Inhibition of aldehyde dehydrogenase and retinoid signaling induces the expansion of human hematopoietic stem cells. Proc Natl Acad Sci USA 103: 11707-11712, 2006.

10. Armstrong L, Stojkovic M, Dimmick I, Ahmad S, Stojkovic P, Hole $\mathrm{N}$ and Lako M: Phenotypic characterization of murine primitive hematopoietic progenitor cells isolated on basis of aldehyde dehydrogenase activity. Stem Cells 22: 1142-1151, 2004.

11. Hess DA, Wirthlin L, Craft TP, Herrbrich PE, Hohm SA Lahey R, Eades WC, Creer MH and Nolta JA: Selection based on CD133 and high aldehyde dehydrogenase activity isolates long-term reconstituting human hematopoietic stem cells. Blood 107: 2162-2169, 2006.
12. Nogami T, Shien T, Tanaka T, Nishiyama K, Mizoo T, Iwamto T, Ikeda H, Taira N, Doihara H and Miyoshi S: Expression of ALDH1 in axillary lymph node metastases is a prognostic factor of poor clinical outcome in breast cancer patients with 1-3 lymph node metastases. Breast Cancer 21: 58-65, 2014.

13. Patel M, Lu L, Zander DS, Sreerama L, Coco D and Moreb JS: ALDH1A1 and ALDH3A1b expression in lung cancers: Correlation with histologic type and potential precursors. Lung Cancer 59: 340-349, 2008.

14. Zhou F, Mu YD, Liang J, Liu ZX, Chen HS and Zhang JF: Expression and prognostic values of tumor stem cell markers ALDH1 and CD133 in colorectal carcinoma. Oncol Lett 7: 507-512, 2014.

15. Grimason AM, Smith HV, Parker JFW, Bukhari Z, Campbell AT and Robertson LJ: Application of DAPI and immunofluorescence for enhanced identification of Cryptosporidium spp oocysts in water samples. Water Res 28: 733-736, 1994.

16. Leiker M, Suzuki G, Iyer VS, Canty JM Jr and Lee T: Assessment of a nuclear affinity labeling method for tracking implanted mesenchymal stem cells. Cell Transplant 17: 911-922, 2008.

17. Ocarino NM, Bozzi A, Pereira RD, Breyner NM, Silva VL, Castanheira P, Goes AM and Serakides R: Behavior of mesenchymal stem cells stained with 4', 6-diamidino-2-phenylindole dihydrochloride (DAPI) in osteogenic and non osteogenic cultures. Biocell 32: 175-183, 2008.

18. Van Vlierberghe RL, Sandel MH, Prins FA, van Iersel LB, van de Velde CJ, Tollenaar RA and Kuppen PJ: Four-color staining combining fluorescence and brightfield microscopy for simultaneous immune cell phenotyping and localization in tumor tissue sections. Microsc Res Tech 67: 15-21, 2005.

19. Huang EH, Hynes MJ, Zhang T, Ginestier C, Dontu G, Appelman H, Fields JZ, Wicha MS and Boman BM: Aldehyde dehydrogenase 1 is a marker for normal and malignant human colonic stem cells (SC) and tracks SC overpopulation during colon tumorigenesis. Cancer Res 69: 3382-3389, 2009.

20. National Comprehensive Cancer Network. (NCCN) clinical practice guidelines in oncology. Esophageal and esophagogastric junction cancers, Version 2, 2017.

21. Xu Y, Chen Q, Yu X, Zhou X, Zheng X and Mao W: Factors influencing the risk of recurrence in patients with esophageal carcinoma treated with surgery: A single institution analysis consisting of 1002 cases. Oncol Lett 5: 185-190, 2013.

22. Rubenstein JH and Chen JW: Epidemiology of gastroesophageal reflux disease. Gastroenterol Clin North Am 43: 1-14, 2014.

23. Li S, Jiang S, Jiang W, Zhou Y, Shen XY, Luo T, Kong LP and Wang HQ: Anticancer effects of crocetin in human esophageal squamous cell carcinoma KYSE-150 cells. Oncol Lett 9: 1254-1260, 2015.

24. Boman BM and Wicha MS: Cancer stem cells: A step toward the cure. J Clin Oncol 26: 2795-2799, 2008 